\title{
Reliable Sideslip Angle Estimation of Four-Wheel Independent Drive Electric Vehicle by Information Iteration and Fusion
}

\author{
Te Chen $\left(\mathbb{D},{ }^{1}\right.$ Long Chen $\left(\mathbb{D},{ }^{1,2}\right.$ Xing Xu $\left(\mathbb{D},{ }^{1,2}\right.$ Yingfeng Cai, ${ }^{1,2}$ \\ Haobin Jiang $\oplus^{1,2}$ and Xiaoqiang Sun ${ }^{1,2}$ \\ ${ }^{1}$ School of Automotive and Traffic Engineering, Jiangsu University, Zhenjiang 212013, China \\ ${ }^{2}$ Automotive Engineering Research Institute, Jiangsu University, Zhenjiang 212013, China \\ Correspondence should be addressed to Long Chen; chenlong@ujs.edu.cn
}

Received 31 December 2017; Accepted 5 March 2018; Published 11 April 2018

Academic Editor: Gisella Tomasini

Copyright (C) 2018 Te Chen et al. This is an open access article distributed under the Creative Commons Attribution License, which permits unrestricted use, distribution, and reproduction in any medium, provided the original work is properly cited.

Accurate estimation of longitudinal force and sideslip angle is significant to stability control of four-wheel independent driven electric vehicle. The observer design problem for the longitudinal force and sideslip angle estimation is investigated in this work. The electric driving wheel model is introduced into the longitudinal force estimation, considering the longitudinal force is the unknown input of the system, the proportional integral observer is applied to restructure the differential equation of longitudinal force, and the extended Kalman filter is utilized to estimate the unbiased longitudinal force. Using the estimated longitudinal force, considering the unknown disturbances and uncertainties of vehicle model, the robust sideslip angle estimator is proposed based on vehicle dynamics model. Moreover, the recursive least squares algorithm with forgetting factor is applied to vehicle state estimation based on the vehicle kinematics model. In order to integrate the advantages of the dynamics-model-based observer and kinematicsmodel-based observer and improve adaptability of observer system in complex working conditions, a vehicle sideslip angle fusion estimation strategy is proposed. The simulations and experiments are implemented and the performance of proposed estimation method is validated.

\section{Introduction}

With the development of automotive technology, drive mode of electric vehicles (EVs) is also experiencing many changes and innovation [1-4]. Emerging four-wheel independent drive electric vehicles (4WID-EVs), as a potential means of future transportation with the controllability of accurate and independent torque, have proven fruitful in energysaving and advanced vehicle motion control and attracted the attention of both industrial and academic communities [57]. 4WID-EVs possess lots of advantages in vehicle dynamics control, such as DYC, in which the accurate estimation of sideslip angle is significant to control performance $[8,9]$. Recently, the increasing requirements of safety and stability in automotive technology promote the rapid growth and all the attendant publicity of intelligent transportation [10-12]. Autonomous ground vehicles and unmanned driving have the great advantages containing higher security, better road utilization, and greatly reduced mobility costs. With regard to the path-following control, the real time and reliable vehicle states estimation contribute to better control performance. Therefore, owning to the consideration that some vehicle states are hard and costly to obtain, the design of model-based vehicle state estimator is essential to estimate the vehicle state via low-cost sensor measurements.

During the past decade, there have been many researches engaged in the field of longitudinal force and sideslip angle estimation. The common algorithm applied in estimation can be summarized as Kalman filter [13-17], nonlinear observer [18-22], optimal estimation method [23-27], information fusion estimation method [15, 17, 28-31], and robust estimation method [32,33], and so on. The core technology in most previous approaches concentrates on solving the estimation problem in complex and limited vehicle running conditions, such as high speed [14, 15], low adhesion [16, 20,34], vehicle parameters time-varying $[29,32]$, and unknown or nonlinear 
disturbances [22-24]. In most prior studies, the longitudinal force observer is designed for traditional internal combustion engine vehicle, and the longitudinal force estimation for EVs especially for 4WID-EVs is still relatively hard to see. In [35], the longitudinal force was calculated by means of integrating the rotational dynamics differential equation of driving wheel, but the influence of noise was accumulated. In 4WID-EVs, the electric driving wheel composed of in-wheel motor and tire is an independent information unit; if we make full use of the measurements of low-cost sensors, such as the current, speed, and bus voltage, the longitudinal force will be achieved cheaply and precisely. Seldom studies have introduced this concept into longitudinal force estimation. For vehicle lateral stability and path-following control, the sideslip angle is an indispensable parameter, and there exists a lot of fruitful progress for sideslip angle estimation. Li et al. presented an adaptive sideslip angle estimator using low-cost sensors in which the multiple model-based Kalman filter was designed to improve the accuracy of estimation [31]. Yoon and Peng estimated the sideslip angle via the combination of the measurements of a global positioning system, an inertial measurement unit, and a magnetometer; the dual Kalman filter was designed to improve the accuracy of estimation via the redundancy of measurements [33].

In this paper, a novel and reliable method of longitudinal force and sideslip angle estimation is proposed for 4WIDEVs. Considering the electric driving wheel model (EDWM) is an uncertain system with unknown input, we utilize the proportional integral observer (PIO) to establish the differential equation of longitudinal force, and the extend Kalman filter (EKF) is introduced to achieve the unbiased estimation of longitudinal force; this design provides a novel thinking of the longitudinal force estimation in 4WID-EVs. In the sideslip angle estimation, the longitudinal forces estimated by longitudinal force observers (LFOs) are regarded and used as the pseudo measurements. The unknown disturbances and uncertainties of vehicle model are considered; the robust sideslip angle estimator is devised on the basis of vehicle dynamics model in which the theoretical robust performance is verified. Furthermore, a recursive least squares method with forgetting factor is used for vehicle state estimation based on the vehicle kinematics model. Then, a vehicle sideslip angle fusion estimation strategy is proposed, in which the accuracy, reliability, and adaptability for the complex working conditions of sideslip angle estimation are improved by the information iteration and fusion between the multimodel-based observers.

The rest of this paper is organized as follows. The vehicle model is presented is Section 2. The vehicle sideslip angle fusion estimation strategy is designed in Section 3. The simulation results are provided in Section 4. The experimental verifications are presented in Section 5, followed by the conclusive remarks.

\section{Vehicle Model}

2.1. Vehicle Dynamics Model. In this section, a two-degree-offreedom (2-DOF) vehicle model in longitudinal and lateral

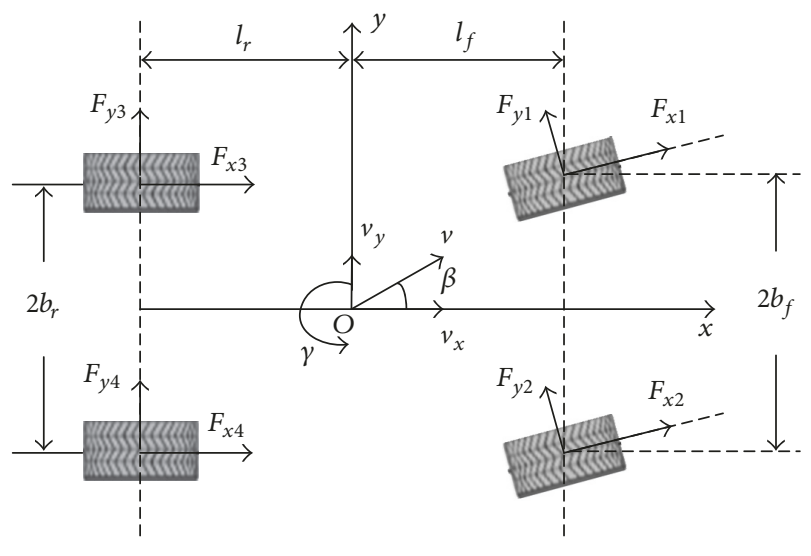

Figure 1: Vehicle dynamics model.

directions is introduced to characterize the vehicle motion; the schematic diagram of vehicle dynamic model is shown in Figure 1. The origin of dynamic coordinate system xoy fixed on the vehicle coincides with the vehicle gravity center; the $x$-axis is the longitudinal axis of the vehicle (the forward direction is positive); the $y$-axis is the lateral axis of the vehicle (the right-to-left direction is positive). The pitch, roll, and vertical motions and the suspension system of the vehicle are ignored. It is assumed that the mechanical properties of each tire are the same. The serial numbers $1,2,3$, and 4 of the wheels are, respectively, corresponding to the frontleft, the front-right, the rear-left, and the rear-right wheel. Assuming that the front wheel steering angle is small, the lateral dynamics equation of the four-wheel vehicle model can be expressed as

$$
\begin{aligned}
m v_{x} \dot{\beta} & =F_{y f}+F_{y r}-m v_{x} \gamma, \\
I_{z} \dot{\gamma} & =l_{f} F_{y f}-l_{r} F_{y r}+\Delta M_{z},
\end{aligned}
$$

where $v_{x}$ and $v_{y}$ represent the longitudinal and lateral vehicle speed at the center of gravity (COG), $\gamma$ is the yaw rate which represents the angular velocity of vehicle at the COG, $\beta$ denotes the sideslip of vehicle at the COG, $m$ represents the vehicle mass, and $I_{z}$ stands for the moment of inertia. $l_{f}$ and $l_{r}$ are the distances from vehicle gravity center to the front and rear axle, respectively. $F_{y f}$ and $F_{y r}$ are the generalized front and rear lateral forces, respectively; that is, $F_{y f}=F_{y 1}+F_{y 2}$, $F_{y r}=F_{y 3}+F_{y 4} \cdot \Delta M_{z}$ is the external yaw moment generated by four in-wheel motors; it can be expressed as

$$
\begin{aligned}
\Delta M_{z}= & \left(F_{x 2}-F_{x 1}\right) b_{f} \cos \delta+\left(F_{x 1}+F_{x 2}\right) l_{f} \sin \delta \\
& +\left(F_{x 4}-F_{x 3}\right) b_{r},
\end{aligned}
$$

where $b_{f, r}$ is the half tread of wheel base, $\delta$ is the steering angle of the front wheels, and $F_{x j}(j=1,2,3,4)$ represents the longitudinal force of the $i$ th tire. The lateral tire force $F_{y f}$ and force $F_{y r}$ can be written as

$$
\begin{aligned}
& F_{y f}=C_{f} \alpha_{f}, \\
& F_{y r}=C_{r} \alpha_{r},
\end{aligned}
$$


where $C_{f}$ and $C_{r}$ are the generalized cornering stiffness of the front and rear tires, respectively. The tire slip angles of front and rear tires can be given by

$$
\begin{aligned}
& \alpha_{f}=\delta_{f}-\frac{l_{f} \gamma}{v_{x}}-\beta \\
& \alpha_{r}=\frac{l_{r} \gamma}{v_{x}}-\beta .
\end{aligned}
$$

The vehicle dynamics model can be derived as

$$
\dot{x}=A x+B u,
$$

where

$$
\begin{aligned}
x & =\left[\begin{array}{ll}
\beta & \gamma
\end{array}\right]^{T}, \\
u & =\left[\begin{array}{ll}
\delta & \Delta M_{z}
\end{array}\right]^{T}, \\
A & =\left[\begin{array}{cc}
-\frac{C_{f}+C_{r}}{m v_{x}} & -1-\frac{l_{f} C_{f}-l_{r} C_{r}}{m v_{x}^{2}} \\
\frac{l_{r} C_{r}-l_{f} C_{f}}{I_{z}} & -\frac{l_{f}^{2} C_{f}+l_{r}^{2} C_{r}}{v_{x} I_{z}}
\end{array}\right], \\
B & =\left[\begin{array}{ll}
\frac{C_{f}}{m v_{x}} & 0 \\
\frac{l_{f} C_{f}}{I_{z}} & \frac{1}{I_{z}}
\end{array}\right] .
\end{aligned}
$$

2.2. EDWM. Each wheel of the FWID-EV is driven by an inwheel motor. And the driving wheel consisting of in-wheel motor and tire is an independent driving and informative unit. As shown in Figure 2, the concept of the EDMW is proposed in this study. The rotational dynamic equation of each wheel can be written as

$$
J_{1} \dot{\omega}_{j}=T_{L j}-F_{x j} r,
$$

where $\omega_{j}$ is the wheel speed of the $j$ th wheel, $J_{1}$ is the wheel moment of inertia, $r$ is the wheel effective rolling radius, and $T_{L j}$ is the load torque of in-wheel motor. The torque balance equation of the output shaft in in-wheel motor can be given by

$$
J_{2} \dot{\omega}_{j}+b \omega_{j}=K_{t} i_{j}-T_{L j},
$$

where $J_{2}$ is the rotational inertia of in-wheel motor rotor, $b$ is the damping coefficient, $K_{t}$ is the motor torque constant, and $i_{j}$ is the bus current. The dynamic voltage balance equation of equivalent circuit in in-wheel motor can be modeled as

$$
u_{j}=R i_{j}+L i_{j}+K_{a} \omega_{j}
$$

where $u_{j}$ is the bus voltage, $R$ is the equivalent resistance of winding, $L$ is the equivalent inductance of winding, and $K_{a}$ is the inverse electromotive force coefficient.

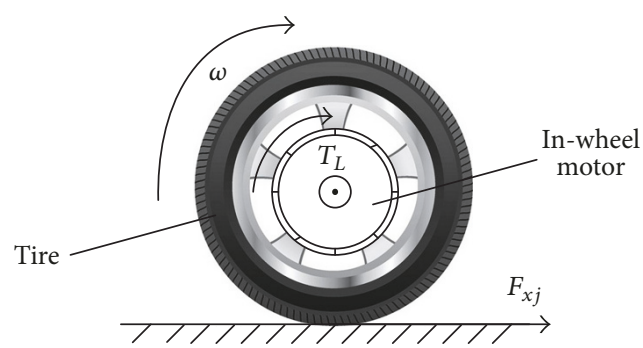

FIgURE 2: Electric driving wheel model.

2.3. Wheel Speed Coupling Relationship. The rotational speed of four wheels can be expressed as

$$
\begin{aligned}
& \omega_{1}=\frac{\left[\left(v_{x}+\gamma b_{f}\right) \cos \delta+\left(v_{y}+\gamma l_{f}\right) \sin \delta\right]}{r}, \\
& \omega_{2}=\frac{\left[\left(v_{x}-\gamma b_{f}\right) \cos \delta+\left(v_{y}+\gamma l_{f}\right) \sin \delta\right]}{r} \\
& \omega_{3}=\frac{v_{x}+\gamma b_{r}}{r} \\
& \omega_{4}=\frac{v_{x}-\gamma b_{r}}{r},
\end{aligned}
$$

where $n_{1}, n_{2}, n_{3}$, and $n_{4}$ represent the corresponding wheel speeds, respectively. $r$ is the effective wheel radius.

\section{Vehicle Sideslip Angle Fusion Estimation Strategy}

\subsection{Dynamics-Based Sideslip Angle Robust Estimation}

3.1.1. Design of LFO. By substituting (7) into (8) and combining it with (9), we have

$$
\begin{aligned}
& \dot{i_{j}}=-\frac{R}{L} i_{j}-\frac{K_{a}}{L} \omega_{j}+\frac{1}{L} u_{j}, \\
& \dot{\omega}_{j}=\frac{K_{t}}{J} i_{j}-\frac{b}{J} \omega_{j}-\frac{r}{J} F_{x j},
\end{aligned}
$$

where $J=J_{1}+J_{2}$. The electric driving wheel model (EDWM) is obtained as

$$
\begin{aligned}
& \dot{x_{w}}=A_{w} x_{w}+B_{w} u_{w}+D_{w} d+E_{w} w_{1}, \\
& y_{w}=C_{w} x_{w}+F_{w} w_{2},
\end{aligned}
$$

where $x_{w}, u_{w}, d$, and $y_{w}$ are the state vector, the known input vector, and the unknown input vector, and measurement vector, respectively. $w_{1}$ and $w_{2}$ are uncorrelated zero mean white noise sequences. The known input and unknown input represent the voltage and longitudinal force, respectively. And

$$
\begin{aligned}
x_{w} & =\left[i_{j} \omega_{j}\right]^{T}, \\
A_{w} & =\left[\begin{array}{rr}
-\frac{R}{L} & -\frac{K_{a}}{L} \\
\frac{K_{t}}{J} & -\frac{b}{J}
\end{array}\right],
\end{aligned}
$$




$$
\begin{aligned}
& B_{w}=\left[\begin{array}{c}
\frac{1}{L} \\
0
\end{array}\right], \\
& D_{w}=\left[\begin{array}{c}
0 \\
r \\
-\frac{J}{J}
\end{array}\right], \\
& C_{w}=\left[\begin{array}{ll}
1 & 0 \\
0 & 1
\end{array}\right], \\
& E_{w}=F_{w}=\left[\begin{array}{l}
1 \\
1
\end{array}\right] .
\end{aligned}
$$

Owing to the existence of unknown input in system (12), the Luenberger observer is not appropriate for the precise state estimation. Consequently, the proportional integral observer (PIO) is utilized to estimate the longitudinal force:

$$
\begin{aligned}
\dot{\widehat{x_{w}}} & =A_{w} \widehat{x_{w}}+B_{w} u_{w}+K_{p}(y-\widehat{y})+D_{w} \widehat{d}+E_{w} w_{1}, \\
\dot{\hat{d}} & =K_{i}\left(y_{w}-\widehat{y_{w}}\right), \\
\widehat{y_{w}} & =C_{w} \widehat{x_{w}}+F_{w} w_{2},
\end{aligned}
$$

where $\widehat{x_{w}}$ is the state estimation, $\widehat{d}$ is the unknown input estimation, $K_{p}$ is the gain matrix of PIO, and $K_{i}$ is the integral matrix of the unknown input estimation. The further form of equation (14) can be derived as

$$
\begin{aligned}
\dot{\hat{x_{w}}}= & \left(A_{w}-K_{p} C_{w}\right) \widehat{x_{w}}+B_{w} u_{w}+K_{p} y_{w}+D_{w} \widehat{d} \\
& +E_{w} w_{1}-K_{p} F_{w} w_{2}, \\
\dot{\hat{d}}= & -K_{i} C_{w} \widehat{x_{w}}+K_{i} y_{w}-K_{i} F_{w} w_{2} .
\end{aligned}
$$

Defining the state estimation error as $e_{w}=\widehat{x_{w}}-x_{w}$, we get

$$
\begin{aligned}
\dot{e_{w}} & =\dot{\overline{x_{w}}}-\dot{x_{w}} \\
& =\left(A_{w}-K_{p} C_{w}\right) e_{w}+D_{w}(\hat{d}-d)-K_{p} F_{w} w_{2} .
\end{aligned}
$$

It can be deduced as

$$
\left[\begin{array}{c}
\dot{e_{w}} \\
\dot{\hat{d}}
\end{array}\right]=A_{w e}\left[\begin{array}{c}
e_{w} \\
\widehat{d}
\end{array}\right]+\left[\begin{array}{c}
D_{w} \\
0
\end{array}\right] d-\left[\begin{array}{c}
K_{p} \\
K_{i}
\end{array}\right] F_{w} w_{2},
$$

where

$$
A_{w e}=\left[\begin{array}{cc}
A_{w}-K_{p} C_{w} & D_{w} \\
-K_{i} C_{w} & 0
\end{array}\right]
$$

Lemma 1 (see [36]). If $-\infty<\lim _{t \rightarrow \infty} d(t)<+\infty$, there exists a PIO for system (11) to guarantee $\lim _{t \rightarrow \infty}(\widehat{x}-x)=0$ for any initial states $x_{w}(0), \widehat{x_{w}}(0)$, and $\widehat{d}(0)$ when $\left(A_{w}, C_{w}\right)$ is observable and $\operatorname{rank}\left\{\left[\begin{array}{cc}A_{w} & D_{w} \\ C_{w} & 0\end{array}\right]\right\}=3$.
Remark 2. Notice that the pole assignment of matrix $A_{w e}$ can be implemented to the specified positions to obtain the gain matrixes $K_{p}$ and $K_{i}$. With the suitable choice of $K_{p}$ and $K_{i}$, the eigenvalues of $A_{w e}$ will locate on the left-half complex plane.

In system (15), the estimation of unknown input is solved by the design of PIO, but the existence of noise will also influence the estimation accuracy. In order to suppress the estimation error caused by noise, the Kalman filter is introduced and combined with PIO for longitudinal force estimation. The logogram of system (15) for Kalman filter design is expressed as

$$
\begin{aligned}
& \dot{x}_{k}(t)=f_{k}\left(x_{k}(t), u_{k}(t)\right)+w_{k 1}(t), \\
& y_{k}(t)=h_{k}\left(x_{k}(t), u_{k}(t)\right)+w_{k 2}(t),
\end{aligned}
$$

where $x_{k}=\left[\begin{array}{ll}\widehat{x_{w}} & \widehat{d}\end{array}\right]^{T}, u_{k}=\left[\begin{array}{ll}u_{w} & y_{w}\end{array}\right]^{T}, y_{k}=\left[\begin{array}{ll}i_{j} & \omega_{j}\end{array}\right]^{T}$.

The steps of EKF are given by the following.

(1) Forecasting Process. Calculate the forecast value

$$
\widehat{x}_{k+1 / k}=\widehat{x}_{k / k}+f\left(\hat{x}_{k / k}\right) T+\frac{A\left(\hat{x}_{k / k}\right) f\left(\hat{x}_{k / k}\right) T^{2}}{2} .
$$

Calculate the variance of prediction error

$$
P_{k+1 / k}=\varphi_{k} P_{k / k} \varphi_{k}^{T}+Q_{k},
$$

where $A\left(x_{k / k}\right)=\left.(\partial f(x) / \partial x)\right|_{x=\widehat{x}_{k / k}}, \varphi_{k}=I+A\left(\widehat{x}_{k / k}\right) T$.

(2) Trimming Process. Calculate the matrix of Kalman gain

$$
K_{k+1}=P_{k+1 / k} H_{k+1}^{T}\left(H_{k+1} P_{k+1 / k} H_{k}^{T}+R_{k+1}\right)^{-1} .
$$

Update the state estimation

$$
\widehat{x}_{k+1 / k+1}=\widehat{x}_{k+1 / k}+K_{k+1}\left[y_{k+1}-h\left(\widehat{x}_{k+1 / k}\right)\right] .
$$

Update the error covariance

$$
P_{k+1 / k+1}=\left(I-K_{k+1} H_{k+1}\right) P_{k+1 / k},
$$

where $H=\partial h(x) /\left.\partial x\right|_{x=\widehat{x}(k / k)}$.

3.1.2. Robust Estimation of Sideslip Angle. As we all know, along with the variation of driving manoeuvre and degree of vehicle stability, the estimation deviations will be influenced by unknown disturbances and uncertainties of vehicle model. For (3), considering the uncertainty of tire cornering stiffness, it can be transformed as $F_{y f}=\left(C_{f}+\Delta C_{f}\right) \alpha_{f}, F_{y r}=\left(C_{r}+\right.$ $\left.\Delta C_{r}\right) \alpha_{r}$, where $\Delta C_{f}$ and $\Delta C_{r}$ express the additional nonlinear factors and are considered to be bounded. In order to weaken the influences caused by the above factors, the Ho-theorybased estimation method is devised in this work for robust sideslip angle estimation.

The generalized dynamic equation of vehicle model (5) for estimation is written as

$$
\begin{aligned}
& \dot{x}=(A+\Delta A) x+B u+D_{1} w_{1}, \\
& y=C x+D_{2} w_{2},
\end{aligned}
$$


where $C=\left[\begin{array}{ll}0 & 1\end{array}\right]^{T}, D_{1}, D_{2}$ are corresponding dimension regular matrixes; $w_{1}$ and $w_{2}$ represent the unknown disturbances and uncertainties. The estimation of LFO is used as the pseudo measurement of vehicle model to calculate $\Delta M_{z}$.

Remark 3. Assume that

(A1) $\left(A, D_{1}\right)$ is controllable and $(A, C)$ is observable; (A2) $D_{2} D_{1}^{T}=0, D_{2} D_{2}^{T}=I$.

Thus, hypothesis $(A 1)$ is the existence condition of the robust observer; hypothesis $(A 2)$ is equivalent to the performance target of the two-type optimal control. Define the form of robust observer as $\hat{x}=\phi(y)$, where $\hat{x}$ is the estimation of $x$. The unbiasedness of estimation means, if there exists arbitrary $\varepsilon$ that satisfies the condition $\varepsilon \leq t$, we have $\widehat{x}(t)=0$.

Remark 4. With bounded $w_{1}$ and $w_{2}$ in (25), the design of robust observer should meet the following condition:

$$
\begin{aligned}
J & =\sup \left\{\frac{\|x-\widehat{x}\|_{2}}{\left\|w_{1,2}\right\|} \mid w_{1,2} \in L_{2}(0, \infty),\left\|w_{1,2}\right\| \neq 0\right\} \\
& <\lambda,
\end{aligned}
$$

where $\lambda(\lambda>0)$ is a given constant. This condition means the design of robust observer makes the energy gain of estimation error $e=x-\widehat{x}$ less than $\lambda$ times of $w_{1}$ and $w_{2}$ to ensure the filter robustness properties.

Remark 5. The robust observer for sideslip angle estimation is proposed as

$$
\dot{\hat{x}}=A \widehat{x}+B u+K(y-C \widehat{x}),
$$

where $K$ is the feedback gain matrix. Combining (25) with (27), we have

$$
\dot{e}=(A-K C) e+\left(D_{1}-K D_{2}\right) w_{1,2} .
$$

Notice that the transfer function between $w_{1,2}$ and $e$ can be given by

$$
G_{w e}(s)=\left[\begin{array}{cc}
A-K C & D_{1}-K D_{2} \\
I & 0
\end{array}\right]
$$

Therefore, performance target of robust estimation can be written as

$$
J=\left\|G_{w e}\right\|_{\infty}<\lambda .
$$

Lemma 6. Defining the strict regular rational transfer functional matrix as

$$
G(s)=\left[\begin{array}{ll}
A & B \\
C & 0
\end{array}\right]
$$

the corresponding Hamiltonian matrix of $G(s)$ is expressed as

$$
H_{G} \stackrel{\text { def }}{=}\left[\begin{array}{cc}
A & \lambda^{-2} B B^{T} \\
-C^{T} C & -A A^{T}
\end{array}\right] .
$$

With regard to system (31), if matrix $A$ is stable, thus the necessary and sufficient condition for $\|G\|_{\infty}<\lambda$ can be expressed as follows: $H_{G} \in \operatorname{dom}($ Ric $), H=\operatorname{Ric}\left(H_{G}\right) \geq 0$, where $H$ is the solution of matrix Riccati equation (introduced in following manuscript).

Lemma 7. With regard to the two Riccati equations

$$
\begin{aligned}
& A^{T} H+H A-H R H+N_{1}=0, \\
& A^{T} H+H A-H R H+N_{2}=0,
\end{aligned}
$$

where $R=R^{T}, Q_{1}=Q_{1}{ }^{T} \geq Q_{2}=Q_{2}{ }^{T} \geq 0$, and $\left(A, Q_{2}{ }^{1 / 2}\right)$ is observable, if $(A, R)$ is steady, that is to say, there exists the solution $\mathrm{H}_{1}>0$ of (33a), then there must exist a solution $\mathrm{H}_{2}$ of (33b) to ensure $H_{1} \geqq H_{2}>0, \operatorname{Re} \lambda\left(A-R H_{2}\right)<0$.

Theorem 8. With regard to the system with disturbances in (25), assuming conditions (A1) and (A2) are satisfied, there exists unbiased estimator to make the Ho norm of transfer function matrix less than $\lambda$. The necessary and sufficient condition of previous description can be stated as follows: if there exists a matrix $H=H^{T}>0$, the establishment-condition of following Riccati equation is satisfied:

$$
A H+H A^{T}+H\left(\lambda^{-2}-C^{T} C\right) H+D_{1} D_{1}^{T}=0,
$$

where the feedback gain matrix is expressed as $K=H C^{T}$.

Proof. Defining $Q=H^{-1}>0$, the premultiplication and postmultiplication to (33a) and (33b) are implemented by matrix $Q$. It can be derived as

$$
Q A+A^{T} Q+\left(\lambda^{-2}-C^{T} C\right)+Q D_{1} D_{1}^{T} Q=0 .
$$

Equation (35) can be converted as

$$
\begin{aligned}
Q(A & \left.-Q^{-1} C^{T} C\right)+\left(A-Q^{-1} C^{T} C\right)^{T} Q+\lambda^{-2} \\
& +Q\left(D_{1}-Q^{-1} C^{T} D_{2}\right)\left(D_{1}-Q^{-1} C^{T} D_{2}\right)^{T} Q \\
= & 0 .
\end{aligned}
$$

The Hamiltonian matrix of (36) is expressed as

$$
\begin{aligned}
& H_{1} \\
& =\left[\begin{array}{cc}
A-Q^{-1} C^{T} C & \left(D_{1}-Q^{-1} C^{T} D_{2}\right)\left(D_{1}-Q^{-1} C^{T} D_{2}\right)^{T} \\
-\lambda^{-2} & -\left(A-Q^{-1} C^{T} C\right)^{T}
\end{array}\right] .
\end{aligned}
$$

There exists the solution of the Riccati equation; according to Lemma 6, it can be noticed that

$$
\left\|\left[\begin{array}{cc}
A-Q^{-1} C^{T} C & D_{1}-Q^{-1} C^{T} D_{2} \\
I & 0
\end{array}\right]\right\|_{\infty}<\lambda,
$$

where $A-Q^{-1} C^{T} C$ is stable. Inequality (37) can be rewritten as

$$
\left\|G_{w e}\right\|_{\infty}=\left\|\left[\begin{array}{cc}
A-K C & D_{1}-K D_{2} \\
I & 0
\end{array}\right]\right\|_{\infty}<\lambda .
$$

The sufficiency of $H \infty$ robust performance is proved. 
Owing to the existence of observer gain in (27), the $H \infty$ performance target of robust observer is guaranteed. Referring to Lemma 6, it can be affirmed that the following matrix Riccati equation is tenable.

$$
\begin{aligned}
H(A & -K C)+(A-K C)^{T} H \\
& +\lambda^{-2} H\left(D_{1}-K D_{2}\right)\left(D_{1}-K D_{2}\right)^{T} H+I=0 .
\end{aligned}
$$

On the basis of assumption $(A 1)$ and Lemma 1, one can know that $H=H^{T}>0$. Defining $Q=H^{-1}>0$, the premultiplication and postmultiplication to (40) are implemented by matrix $Q$. It can be derived as

$$
\begin{aligned}
Q(A & -K C)^{T}+(A-K C) Q \\
& +\lambda^{-2}\left(D_{1}-K D_{2}\right)\left(D_{1}-K D_{2}\right)^{T}+Q Q=0
\end{aligned}
$$

Equation (41) can be converted as

$$
\begin{gathered}
\lambda^{2} Q A^{T}+\lambda^{2} A Q+\lambda^{2} Q\left(I-\lambda^{2} C^{T} C\right) Q+D_{1} D_{1}^{T} \\
+\left(K-\lambda^{2} Q C^{T}\right)\left(K-\lambda^{2} Q C^{T}\right)^{T}=0 .
\end{gathered}
$$

On the basis of assumption $(A 1)$ and Lemma 7, one can know that there must exist a solution $H\left(\lambda^{2} Q \geqq H=H^{T}>0\right)$ to ensure the following matrix Riccati equation is tenable:

$$
H A^{T}+A H+H\left(\lambda^{-2}-C^{T} C\right) H+D_{1} D_{1}^{T}=0
$$

The necessity of $H \infty$ robust performance is proved. The estimated sideslip angle is denoted as $\beta_{H}$.

3.2. Kinematics-Based Vehicle State Observer Design. The observation equation of wheel speed coupling relationship system in (10) is written as

$$
Y=H \xi+V
$$

where $Y$ is the observation vector, $\xi$ is the state to be estimated, $H$ is the measurement matrix, and $V$ is the zero mean white noise sequence. The corresponding vector and matrix are represented as

$$
\begin{aligned}
Y & =\left[\begin{array}{llll}
\omega_{1} & \omega_{2} & \omega_{3} & \omega_{4}
\end{array}\right]^{T}, \\
H & =\left[\begin{array}{ccc}
\frac{\cos \delta}{r} & \frac{\sin \delta}{r} & \frac{b_{f} \cos \delta+l_{f} \sin \delta}{r} \\
\frac{\cos \delta}{r} & \frac{\sin \delta}{r} & \frac{-b_{f} \cos \delta+l_{f} \sin \delta}{r} \\
\frac{1}{r} & 0 & \frac{b_{r}}{r} \\
\frac{1}{r} & 0 & -\frac{b_{r}}{r}
\end{array}\right], \\
\xi & =\left[\begin{array}{lll}
v_{x} & v_{y} & \gamma
\end{array}\right]^{T} .
\end{aligned}
$$

According to the characteristics of formula (12), it is known that the least square method (RLS) can be used to estimate the vehicle state like longitudinal speed, lateral speed, and yaw rate. However, in the iteration process, with the increase of data, the least square method will show the phenomenon of data saturation; that is to say, as time goes on, more and more data are collected, and the information provided by the new data is submerged in the ocean of old data. If the estimation algorithm gives the same degree of trust to the old and new data, it will lose the ability of correction. To overcome the phenomenon of data saturation and to track the change of time-varying parameters, the least square method with forgetting factor is applied to vehicle state estimation, and the recursive algorithm of least square method is given by

$$
\begin{aligned}
\hat{\xi}(k) & =\widehat{\xi}(k-1)+K(k)(Y(k)-H(k) \hat{\xi}(k-1)), \\
K(k) & =\frac{P(k-1) H(k)}{\rho+H^{T}(k) P(k-1) H(k)}, \\
P(k) & =\frac{1}{\rho}\left(I-K(k) H^{T}(k)\right) P(k-1),
\end{aligned}
$$

where $K(k)$ is the gain matrix, $P(k)$ is the covariance matrix, and $\rho(0<\rho \leq 1)$ is the forgetting factor used to balance the fast-tracking ability and resisting-disturbance capacity of the estimation results. The estimated vehicle longitudinal speed, lateral speed, and yaw rate are denoted as $v_{x R}, v_{y R}$, and $\gamma_{R}$, respectively.

3.3. Fusion Estimation Strategy. The robust sideslip angle observer in Section 3.1.2 is designed on the basis of linear tire model; it can get accurate estimations in conventional working condition. Although the uncertainty is considered in the observer design process, in some complex conditions, such as when the tire works in strong nonlinear region, the estimation results will deviate to a certain extent. The least square method with forgetting factor in Section 3.2 improves the reliability of vehicle state estimation results in addition to inheriting the advantages of the least square algorithm. But this method relies on accurate wheel speed measurement, and the estimation effect will be affected when the longitudinal slip rate of tire is too large. Consequently, we can improve the accuracy and reliability of vehicle state estimation by the error iteration and information fusion between the multimodelbased observers. The proposed vehicle state fusion estimation strategy is shown in Figure 3.

In (10), theoretically, $\omega_{1}, \omega_{2}, \omega_{3}$, and $\omega_{4}$ show the ideal wheel speed, and it is impossible for ideal wheel speed to totally equal the actual wheel speed. Moreover, if matrix $H$ is an ill-conditioned matrix, little variation may cause the deviation to the solution of RLS. Therefore, considering the deviation caused by the uncertainty of the model in (10), an error compensation mechanism for longitudinal and lateral vehicle speed estimation is developed by PID controller. The estimation error of yaw rate between the real yaw rate and $\gamma_{R}$ is defined as $\Delta \gamma=\gamma_{R}-\gamma$ and used as the input of the PID1 and PID2, the controllers PID1 and PID2 output the corresponding longitudinal and lateral vehicle speed by 


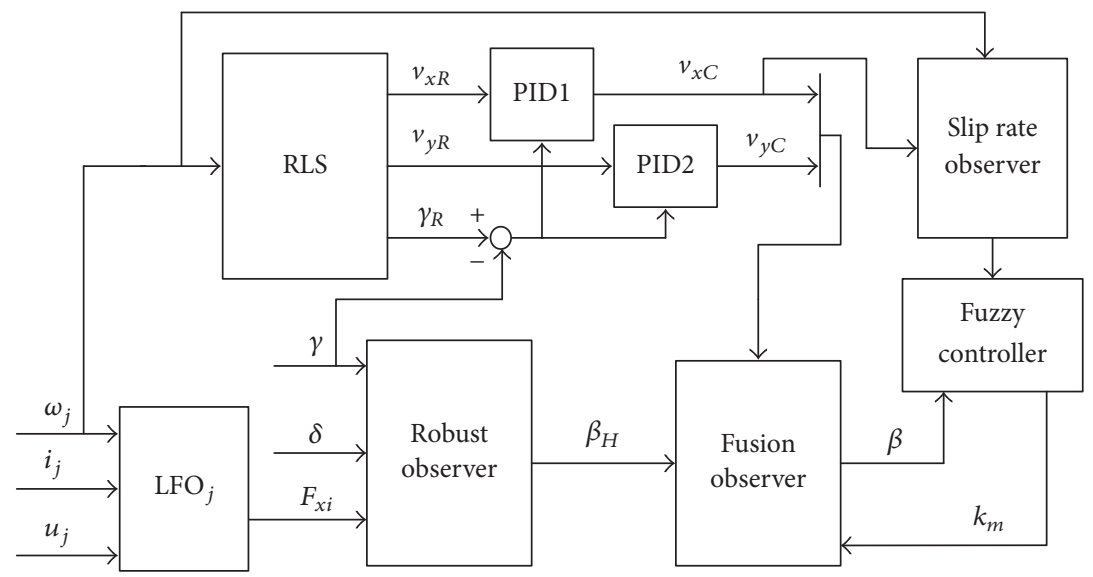

FIgURE 3: Vehicle sideslip angle fusion estimation strategy.
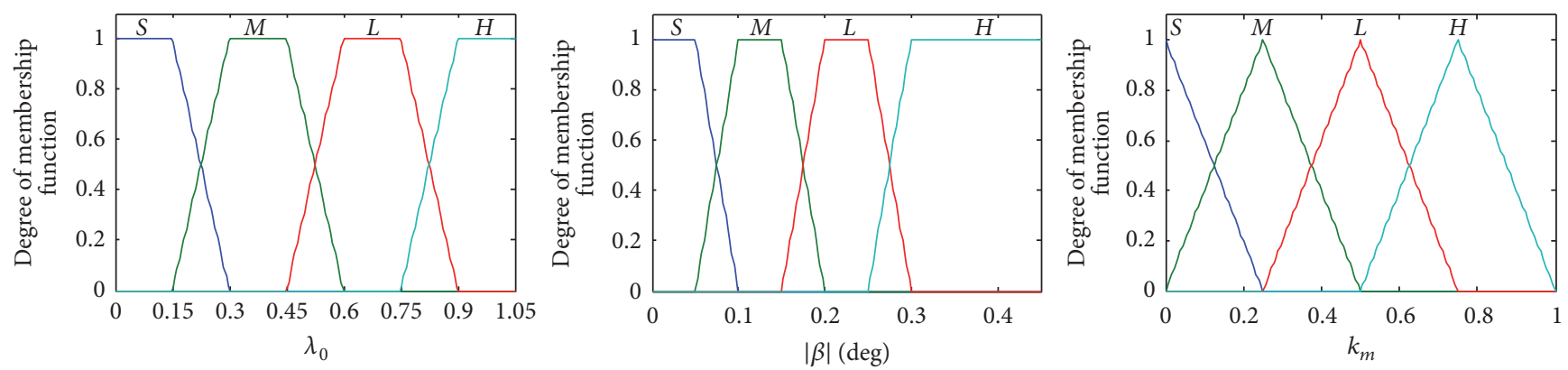

FIgURE 4: Degree of membership functions of $\lambda_{0},|\beta|$, and $k_{m}$.

error compensation, respectively, and they can be expressed as follows (denoted as $v_{x C}$ and $v_{y C}$ ):

$$
\begin{aligned}
& v_{x C}=v_{x R}+k_{\mathrm{PID} 1} \Delta \gamma, \\
& v_{y C}=v_{y R}+k_{\mathrm{PID} 2} \Delta \gamma,
\end{aligned}
$$

where $k_{\mathrm{PID} 1}$ and $k_{\mathrm{PID} 2}$ are the compensation coefficient of controllers PID1 and PID2. Then the kinematics-based sideslip angle estimation is obtained as $\beta_{k}=\arctan \left(v_{x C} / v_{y C}\right)$.

The longitudinal slip rate of tire can be calculated by

$$
\lambda_{j}=\frac{\omega_{j} r-v_{x}}{v_{x}} \quad(j=1,2,3,4) .
$$

In this section, the fuzzy controller is designed to fuse the results of kinematics-based sideslip angle estimation and robust sideslip angle estimation. According to the above analysis, the absolute values of sideslip angle $|\beta|$ and $\lambda_{0}=$ $\left|\lambda_{1}\right|+\left|\lambda_{2}\right|+\left|\lambda_{3}\right|+\left|\lambda_{4}\right|$ are chosen as the inputs of fuzzy controller, in which $|\beta|$ and $\lambda_{0}$ are used to embody the confidence level of the estimation results of $\beta_{k}$ and $\beta_{H}$. When $|\beta|$ is excessively large, the lateral offset of the tire increases and the nonlinear characteristic is enhanced; then it is necessary to reduce the weight of dynamics-based sideslip angle estimation result. When $\lambda_{0}$ is excessively large, it shows that the longitudinal slip is more severe; then it is
TABLE 1: Fuzzy control rules.

\begin{tabular}{cccccc}
\hline$k_{m}$ & & & & $|\beta|$ & \\
& & $S$ & $M$ & $L$ & $H$ \\
\hline & $S$ & $S$ & $M$ & $M$ & $L$ \\
$\lambda_{0}$ & $M$ & $M$ & $L$ & $L$ & $H$ \\
& $L$ & $L$ & $H$ & $H$ & $H$ \\
& $H$ & $L$ & $H$ & $H$ & $H$ \\
\hline
\end{tabular}

necessary to reduce the weight of kinematics-based sideslip angle estimation result. The fuzzy output $k_{m}$ is calculated by fuzzy controller and used as the fusion weight coefficient between $\beta_{k}$ and $\beta_{H}$, and the fusion estimation results of sideslip angle are expressed as

$$
\beta=k_{m} \beta_{k}+\left(1-k_{m}\right) \beta_{H} \text {. }
$$

The membership functions of the input and output in fuzzy controller are shown in Figure 4. The fuzzy control rules are listed in Table 1.

Synchronously, the fusion estimation result of sideslip angle $\beta$ is regarded as the pseudo measurement value and used as the input of fuzzy controller; the compensation estimation result of longitudinal vehicle speed $v_{x C}$ is used as the input of slip rate observer. Thus, both $\beta$ and $v_{x C}$ provide 
TABLE 2: Parameters of vehicle and in-wheel motors.

\begin{tabular}{lcc}
\hline Symbol & Parameters & Value and units \\
\hline$m$ & Vehicle mass & $710 \mathrm{~kg}$ \\
$r$ & Effective radius of wheel & $0.245 \mathrm{~m}$ \\
$l_{f}$ & Distances from vehicle gravity center to the front axle & $0.795 \mathrm{~m}$ \\
$l_{r}$ & Distances from vehicle gravity center to the rear axle & $0.975 \mathrm{~m}$ \\
$b_{f}, b_{r}$ & Half treads of the front (rear) wheels & $0.775 \mathrm{~m}$ \\
$C_{f}$ & Equivalent cornering stiffness of front wheel & $60000 \mathrm{~N} / \mathrm{rad}$ \\
$C_{r}$ & Equivalent cornering stiffness of rear wheel & $40000 \mathrm{~N} / \mathrm{rad}$ \\
$I_{z}$ & Moment of inertia & $1000 \mathrm{~kg} \cdot \mathrm{m}^{2}$ \\
$R$ & Equivalent resistance of winding & $0.688 \Omega$ \\
$K_{a}$ & Inverse electromotive force coefficient & $0.06 \mathrm{Nm} / \mathrm{A}$ \\
$K_{t}$ & Motor torque constant & $11.43 \mathrm{Nm} / \mathrm{A}$ \\
$J$ & Sum of inertia moment of wheel and motor & $7.143 \mathrm{~kg} \cdot \mathrm{m}^{2}$ \\
$b$ & Damping coefficient & $0.643 \mathrm{Nm} \cdot \mathrm{sec} / \mathrm{rad}$ \\
$L$ & Equivalent inductance of winding & $0.125 \mathrm{H}$ \\
\hline
\end{tabular}

the judgement datum for next-step iterative estimation; then the reliability, anti-interference ability, and self-adaptability in multiple working conditions of presented observer system are improved by the error iteration compensation between the multimodel-based observers.

\section{Simulation Results}

To validate the effectiveness of the designed LFO and proposed vehicle sideslip angle fusion estimation strategy in this work, the simulations are carried out on a highfidelity CarSim-Simulink joint simulation platform. CarSim is used to provide the whole vehicle model; the estimation of longitudinal forces and sideslip angle is achieved in Matlab/Simulink. The parameters of vehicle and in-wheel motors are listed in Table 2. In simulation and following road test, the uncertainty of tire stiffness is assumed as $-0.3 C_{f} \leq$ $\Delta C_{f} \leq 0.3 C_{f},-0.3 C_{r} \leq \Delta C_{r} \leq 0.3 C_{r}$. In the robust sideslip angle estimator design, in order to adjust to the constraint conditions of $(A 1)$ and (A2), system (25) is expanded in the form of

$$
\begin{aligned}
& \dot{x}=A x+B u+\left[\begin{array}{ll}
1 & 0 \\
0 & 0
\end{array}\right]\left[\begin{array}{l}
w_{1} \\
w_{2}
\end{array}\right], \\
& y=\left[\begin{array}{ll}
0 & 1
\end{array}\right] x+\left[\begin{array}{ll}
0 & 1
\end{array}\right]\left[\begin{array}{l}
w_{1} \\
w_{2}
\end{array}\right] .
\end{aligned}
$$

4.1. Double Lane Changes (DLC) Manoeuvre. In this case, the DLC manoeuvre, as shown in Figure 5, is carried out. In simulation, the road friction coefficient is set to be 0.6 . The vehicle speed maintains a constant of $20 \mathrm{~m} / \mathrm{s}$.

The designs of LFO for wheels $1,2,3$, and 4 are implemented, respectively, and the estimation results of longitudinal forces are shown in Figure 6. It can be found that the designed LFOs can estimate the longitudinal force accurately with the measurements of currents, speeds, and voltages. The estimation effectiveness of LFOs integrated with PIO and

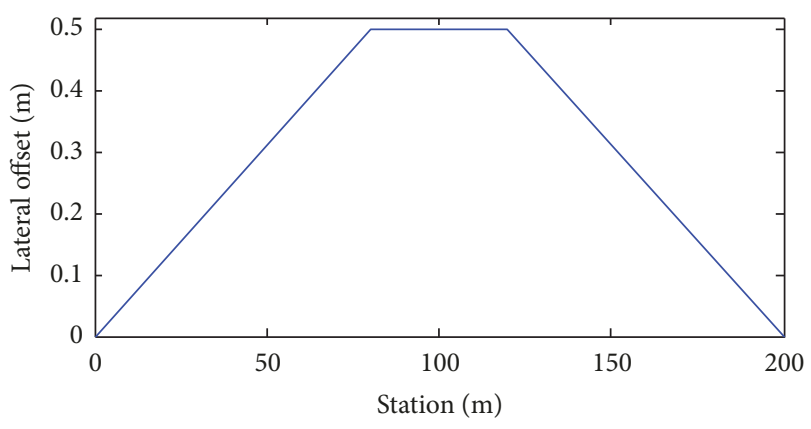

FIGURE 5: DLC manoeuvre.

EKF is better than the one only based on PIO; it means that the design of PIO makes it possible to estimate the unknown input of system (longitudinal forces), and the coalition of EKF reduces the influences of noise and guarantees the estimation accuracy. That is to say, the estimated values of LFOs are reliable to substitute the measurements of sensors as the inputs of robust sideslip angle estimator. In DLC manoeuvre, the vehicle speed is set to be relatively larger, and the vehicle actuating manoeuvre changes violently at the beginning; accordingly there was a certain degree tremble in longitudinal forces, but the proposed LFO still can track the real longitudinal force in real time.

The sideslip angle estimation is shown in Figure 7. It is known that both the robust observer and the RLS with compensation can estimate the vehicle sideslip angle in real time. In the DLC manoeuvre with high speed and low road adhesion, the tire longitudinal slip has a certain influence on the RLS estimation result, and the estimation accuracy of RLS is lower than that of robust observer. And the estimation accuracy of sideslip angle can be effectively improved by using the weighted iterative fusion estimation method. In order to further show the accuracy of proposed estimation method, the root-mean-square (RMS) error $E_{\mathrm{RMS}}$ between real vehicle state and estimated value is used for the 

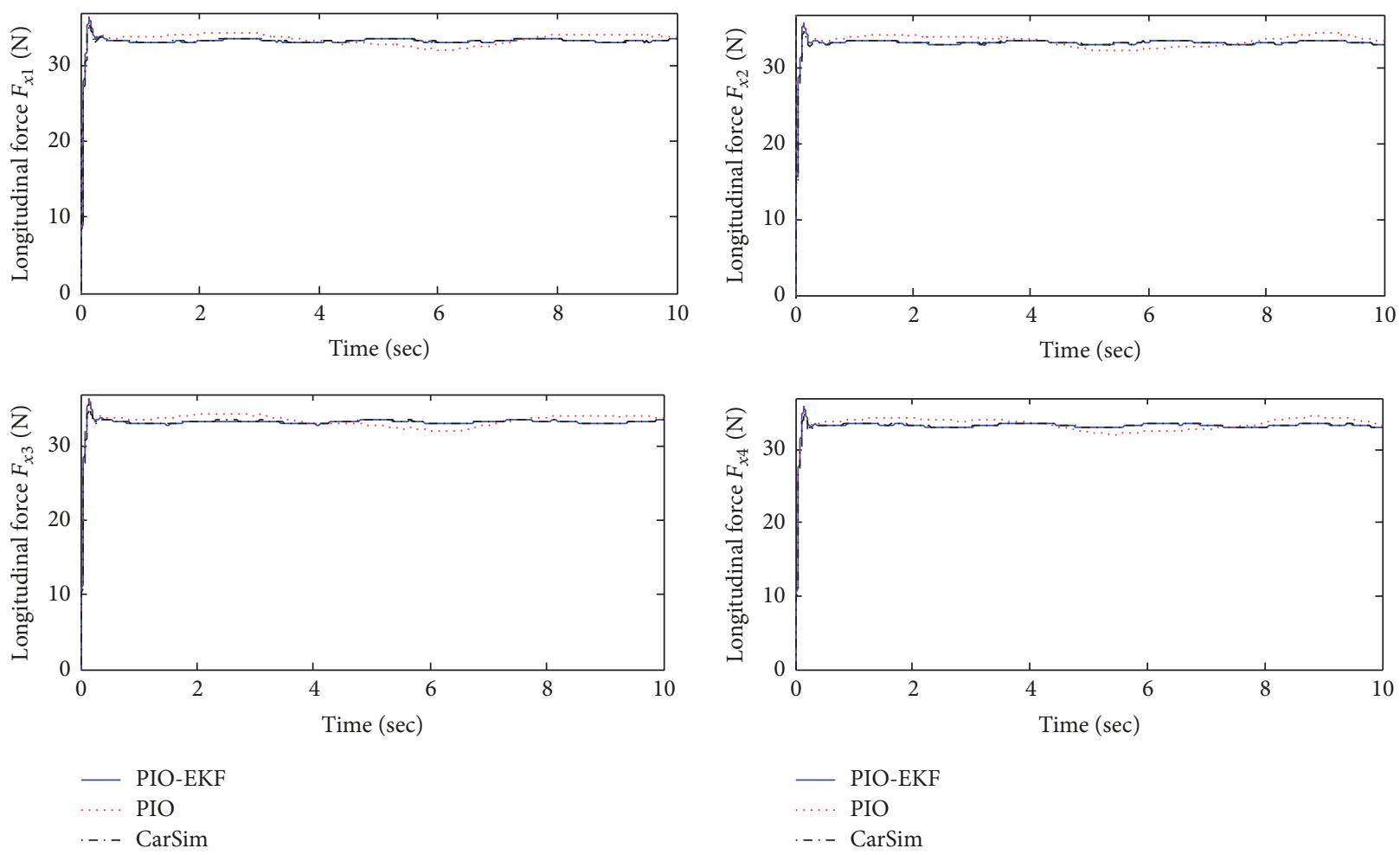

FIgURE 6: Estimation of longitudinal forces.

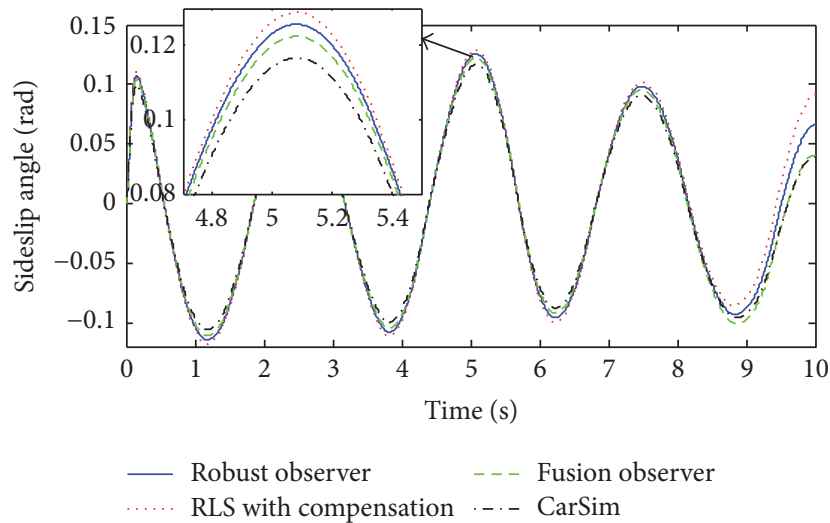

FIGURE 7: Estimation of sideslip angle.

quantitative evaluation and can be computed by the following equation:

$$
E_{\mathrm{RMS}}=\sqrt{\frac{1}{N_{s}} \sum_{i=1}^{N_{s}}\left(\hat{x}_{i}-x_{i}\right)^{2}},
$$

where $N_{s}$ is the number of samples; $x_{i}$ and $\widehat{x}_{i}$ denote the measured and estimated vehicle state at the $i$ th sample. The comparison of $E_{\mathrm{RMS}}$ of vehicle state estimation in DLC manoeuvre simulation is shown in Table 3. In table, the sideslip angle estimation method of robust observer, RLS with compensation, and fusion observer are abbreviated as RO,
RWC and FO. It can be found that $E_{\mathrm{RMS}}$ of proposed longitudinal force estimation method and fusion method are less than that of other estimation methods, which indicates that the proposed method improves the accuracy and reliability of longitudinal force and sideslip angle estimation.

4.2. J-Turn Manoeuvre. In the simulation of J-turn manoeuvre, the road friction coefficient is set to be 1.0; in addition, considering the time-varying characteristic of vehicle speed, a sine wave with the amplitude of 1 and frequency of $1 \mathrm{rad} / \mathrm{s}$ is superimposed on the base speed of $10 \mathrm{~m} / \mathrm{s}$. The corresponding vehicle speed and steering wheel angle in simulation are shown in Figure 8.

The estimation results of longitudinal forces are shown in Figure 9. Similar to the estimation results in DLC manoeuvre, it can be found that the estimation results of longitudinal force based on PIO-EKF show better estimation ability.

As shown in Figure 10, under the rapid steering conditions, the proposed fusion method can still maintain good estimation performance. In the J-turn manoeuvre, owning to the fast-changing steering wheel angle and the time-varying vehicle speed, the vehicle sideslip angle is relatively larger; in this case, the estimation accuracy of the robust observer is lower than RLS with compensation. The presented fusion estimation strategy can integrate the advantages of the two observers effectively, ensure the estimation accuracy, and simultaneously improve the anti-interference performance of estimation system. Moreover, it can be found that the fusion estimation strategy enhances the adaptability and reliability of the estimation system in multiple conditions. Similarly, 
TABLE 3: Comparison of $E_{\mathrm{RMS}}$ in DLC manoeuvre simulation.

\begin{tabular}{lcccc}
\hline & \multicolumn{2}{c}{ Longitudinal force observer } & Observer & \multicolumn{2}{c}{ Sideslip angle observer } \\
& PIO & PIO-EKF & RO & RWC \\
\hline Longitudinal force $F_{x 1}$ & 0.4736 & 0.3284 & $\times$ & $\times$ \\
Longitudinal force $F_{x 2}$ & 0.4654 & 0.3948 & $\times$ & $\times$ \\
Longitudinal force $F_{x 3}$ & 0.4945 & 0.3166 & $\times$ & $\times$ \\
Longitudinal force $F_{x 4}$ & 0.4835 & 0.3154 & $\times$ & $\times$ \\
Sideslip angle & $\times$ & $\times$ & 0.2091 & $\times$ \\
\hline
\end{tabular}
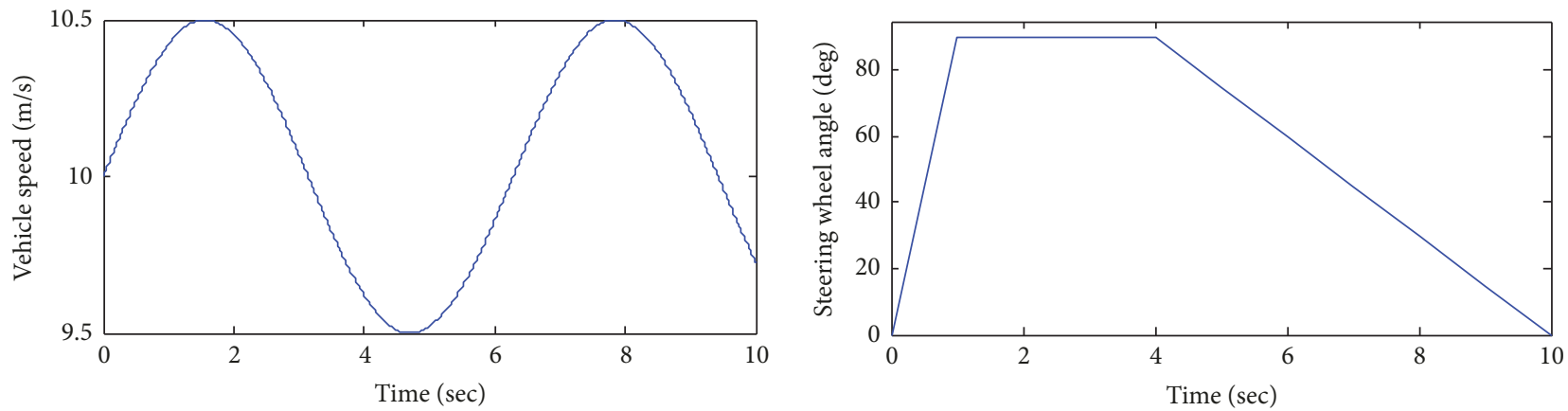

FIGURE 8: Vehicle speed and steering wheel angle.
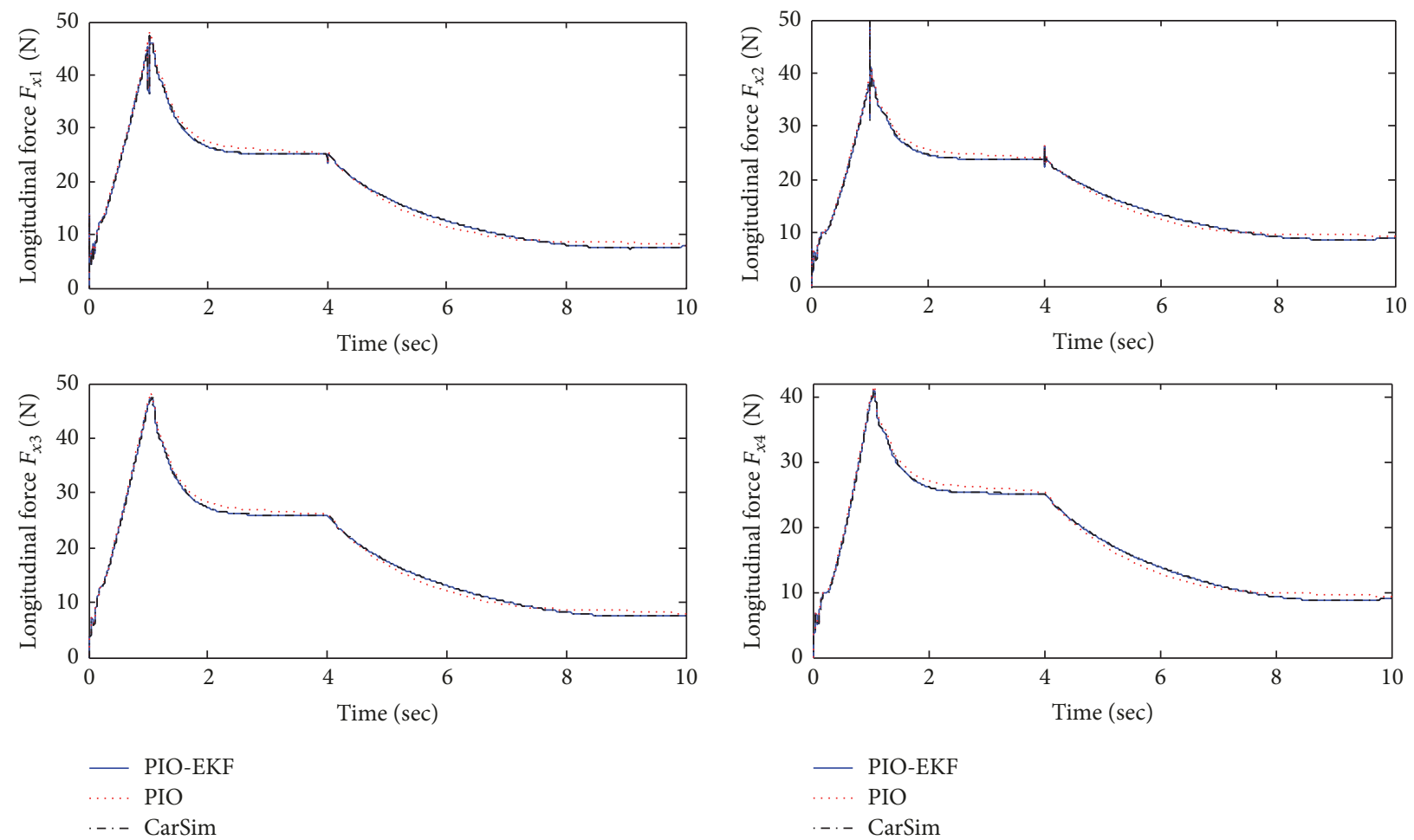

FIgURE 9: Estimation of longitudinal forces. 
TABLE 4: Comparison of $E_{\mathrm{RMS}}$ in J-turn manoeuvre simulation.

\begin{tabular}{lcccc}
\hline & \multicolumn{2}{c}{ Observer } & \multicolumn{2}{c}{ Sideslip angle observer } \\
& PIO & Longitudinal force observer & RO & RWC \\
\hline Longitudinal force $F_{x 1}$ & 0.7678 & PIO-EKF & $\times$ & $\times$ \\
Longitudinal force $F_{x 2}$ & 0.7144 & 0.3346 & $\times$ & $\times$ \\
Longitudinal force $F_{x 3}$ & 0.7513 & 0.3703 & $\times$ & $\times$ \\
Longitudinal force $F_{x 4}$ & 0.7098 & 0.3111 & $\times$ & $\times$ \\
Sideslip angle & $\times$ & 0.3217 & 0.4136 & $\times$ \\
\hline
\end{tabular}

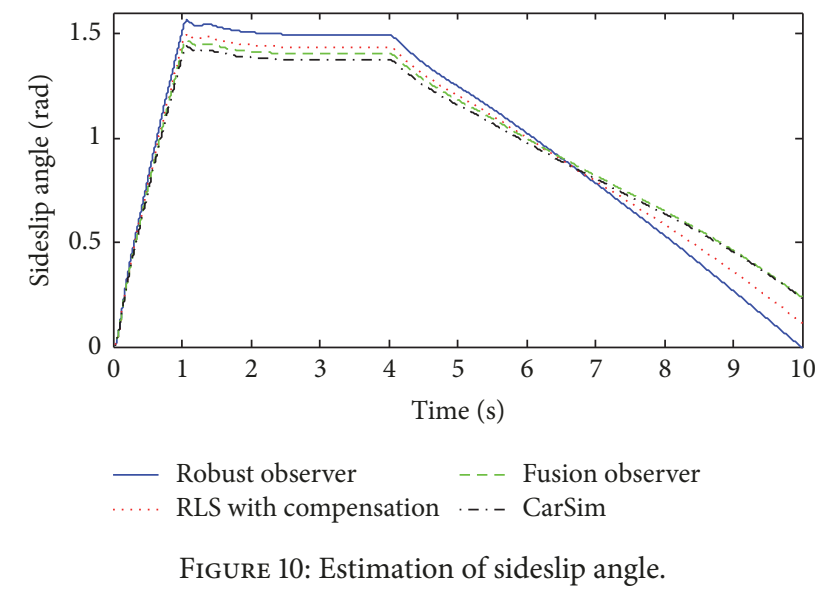

as listed in Table 4, $E_{\mathrm{RMS}}$ in J-turn manoeuvre simulation was computed for further verification of the superiority of the proposed method in improving the estimation accuracy of vehicle state. And the presented longitudinal force and sideslip angle estimation method still maintain better estimation performance.

\section{Experimental Results}

In this section, the experiment is executed for further validation about the estimation performance of proposed estimation method. At present, the sensor for direct longitudinal force measurement is still unavailable on our fourwheel independent drive electric vehicle, so the verification of LFO is implemented on the chassis dynamometer bench. The robust estimator is validated by the experimental data from road test, in which the estimated longitudinal forces by LFOs are regarded and used as the credible pseudo measurements of the fusion estimation system.

5.1. Test on Chassis Dynamometer Bench. The vehicle test on chassis dynamometer bench is shown in Figure 11. The experimental vehicle is a four-wheel independent drive electric vehicle which is refitted from a single-motor drive electric vehicle and actuated by four in-wheel motors. The whole vehicle control system is achieved on a rapid prototyping platform. The currents, speeds, and voltages of in-wheel motors were measured with corresponding sensors and recorded by the host computer through CAN bus. The longitudinal

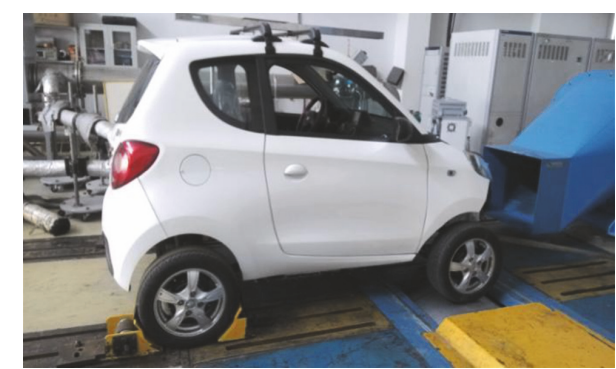

FIGURE 11: Vehicle test on chassis dynamometer bench.

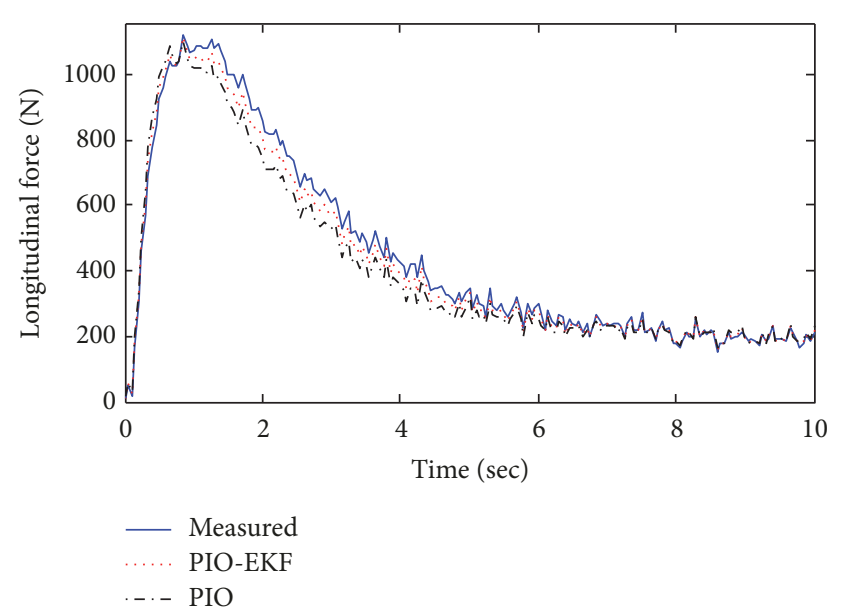

FIgURE 12: Estimation of longitudinal forces.

forces were recorded by the data acquisition system of chassis dynamometer.

In this work, the experimental data of front-right electric driving wheel is selected to testify the proposed LFO; the experimental result is shown in Figure 12. It can be found that there exists some estimation error during the process of acceleration, but the error is finite. The LFO can track the longitudinal force with high accuracy after $4 \mathrm{~s}$ when the driving condition approaches the manoeuvre of uniform speed.

5.2. Road Test. Figure 13(a) shows the photo of vehicle road test. As shown in Figure 13(b), the road test is implemented in the form of serpentine trajectory, 10 traffic piles are placed as 


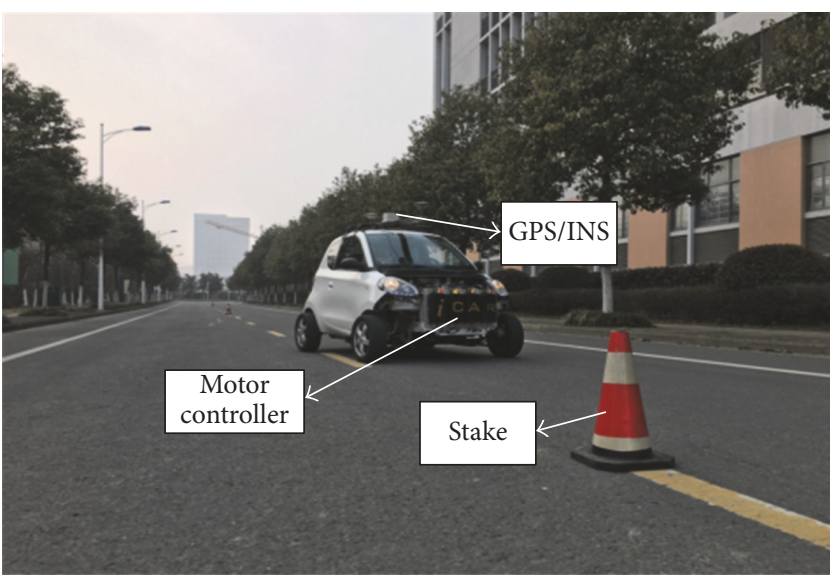

(a) Experimental vehicle

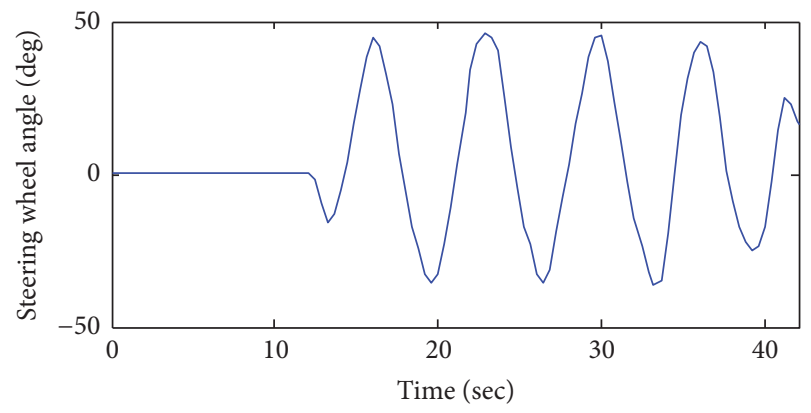

(c) Steering wheel angle

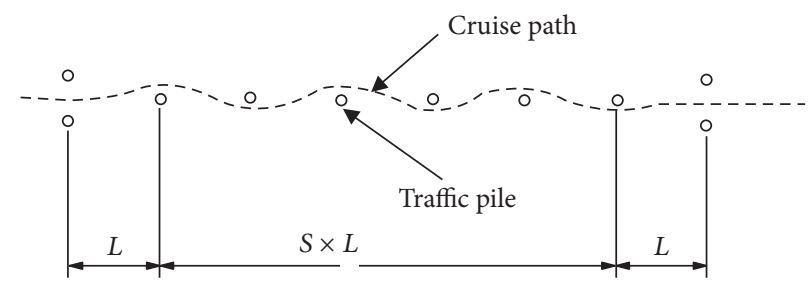

(b) Experimental trajectory

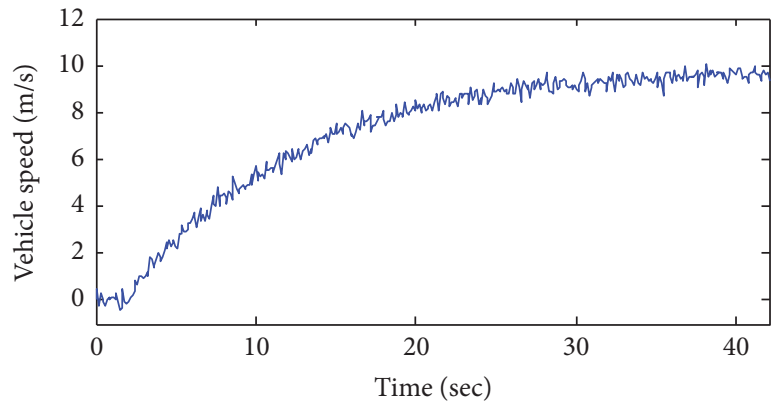

(d) Vehicle speed

FIGURE 13: Road test.

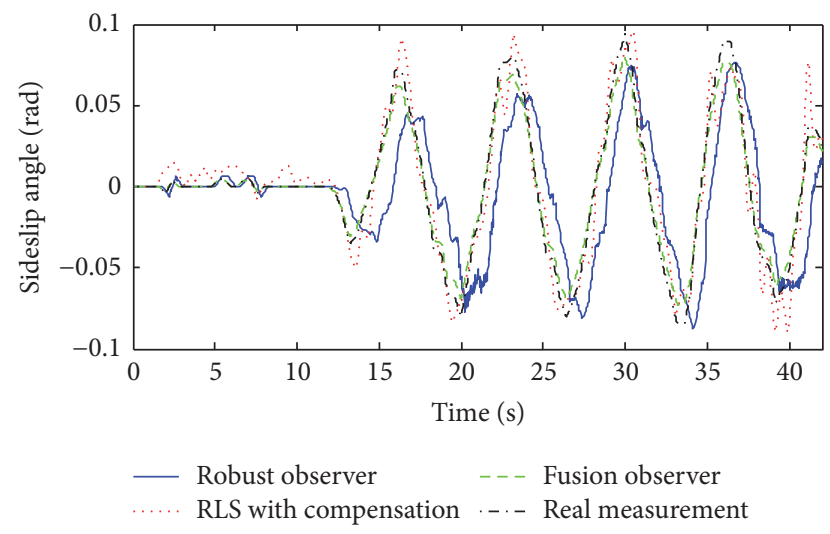

FIGURE 14: Estimation of sideslip angle.

the obstacle, and the vehicle speed cruising is regulated well by a speed controller. The corresponding steering wheel angle and vehicle speed measured in road test are shown in Figures 13(c) and 13(d), respectively. Figure 14 shows the estimation results of sideslip angle. From Figure 14, it can be seen that forgetting-factor-based RLS algorithm with compensation can track the changing trend of sideslip angle in real time; in addition, by contrasting the sideslip angle estimation results, there exists some fluctuation in the estimation of RLS. The reason is that the magnitude of sideslip angle is far less than wheel speed, and small disturbances in wheel speeds may lead to relatively large estimation fluctuations. Even so, the forgetting-factor-based RLS algorithm with compensation is still able to maintain better estimation results, and the range of volatility is relatively acceptable. The robust observer has good estimation accuracy and stability, but the robust observer is designed on the basis of dynamic model, so the real time tracking ability of which is weaker than RLS. The fusion estimation strategy can synthesize the estimated results of the two kinds of observers and obtain optimal sideslip angle estimation. In order to further validate the accuracy of the proposed estimation method in practice, the comparisons of $E_{\mathrm{RMS}}$ in chassis dynamometer bench test and road test are shown in Table 5. Notice that the effect of the estimation method is satisfactory in actual vehicle application.

\section{Conclusion}

This work presented a novel longitudinal force and sideslip angle fusion estimation strategy for 4WID-EVs. The EDWM was established and introduced into the longitudinal force estimation. Considering the longitudinal force is the unknown input of EDWM, the PIO was used to restructure the differential equation of longitudinal force, and the EKF was applied to restrain the influence of noise and improve the accuracy of longitudinal force estimation. Based on 
TABLE 5: Comparison of $E_{\mathrm{RMS}}$ in experiment.

\begin{tabular}{|c|c|c|c|c|c|}
\hline \multirow{3}{*}{$E_{\mathrm{RMS}}$} & \multicolumn{5}{|c|}{ Observer } \\
\hline & \multicolumn{2}{|c|}{ Longitudinal force observer } & \multicolumn{3}{|c|}{ Sideslip angle observer } \\
\hline & $\mathrm{PIO}$ & PIO-EKF & $\mathrm{RO}$ & RWC & $\mathrm{FO}$ \\
\hline Longitudinal force & 1.9374 & 1.1007 & $x$ & $x$ & $x$ \\
\hline Sideslip angle & $\times$ & $x$ & 0.1966 & 0.1379 & 0.0743 \\
\hline
\end{tabular}

the longitudinal force estimation, we proposed a robust sideslip angle estimation method with the consideration of the unknown disturbances and uncertainties of vehicle model, and the theoretical proof of robust estimator was provided. Moreover, a kinematics-based vehicle state observer was developed in which the forgetting-factor-based RLS algorithm with error compensation was applied for sideslip angle estimation. Then fusion estimation strategy was presented for more accurate estimation of sideslip angle by the error compensation and iteration between the multimodel-based observers. The simulations of DLC manoeuvre and J-turn manoeuvre were executed, and the results show that the presented LFO can track the longitudinal force in real time, and the fusion estimation strategy has the satisfactory accuracy in estimation. For further validation, the test on chassis dynamometer bench and road test were implemented; the effectiveness of proposed estimation method was verified.

\section{Conflicts of Interest}

The authors declare that they have no conflicts of interest.

\section{Acknowledgments}

This work was supported by the National Natural Science Foundation of China (Grant nos. U1664258 and U1564201), the "Six Major Talent Project" of Jiangsu Province (Grant no. 2014-JXQC-004), the "333 Project" of Jiangsu Province (Grant no. BRA2016445), the Primary Research \& Development Plan of Jiangsu Province (Grant nos. BE 2017129 and BE2016149), the Natural Science Foundation of Jiangsu Province (Grant no. BK 20160525), and the Natural Science Foundation of Colleges and Universities in Jiangsu (Grant no. 16KJB580012).

\section{References}

[1] R. Wang, H. Zhang, J. Wang, F. Yan, and N. Chen, "Robust lateral motion control of four-wheel independently actuated electric vehicles with tire force saturation consideration," Journal of The Franklin Institute, vol. 352, no. 2, pp. 645-668, 2015.

[2] T. Chen, X. Xu, L. Chen, H. Jiang, Y. Cai, and Y. Li, "Estimation of longitudinal force, lateral vehicle speed and yaw rate for fourwheel independent driven electric vehicles," Mechanical Systems and Signal Processing, vol. 101, pp. 377-388, 2018.

[3] X. Xu, T. Chen, L. Chen, and W. Wang, "Longitudinal force estimation for motorized wheels driving electric vehicle based on improved closed-loop subspace identification," Journal of
Jiangsu University (Natural Science Edition), vol. 37, no. 6, pp. 650-656, 2016.

[4] H. Jiang, A. Li, S. Ma, and L. Chen, "Design and performance analysis of airflow energy recovery device of electric vehicle," Journal of Jiangsu University (Natural Science Edition), vol. 38, no. 2, pp. 125-132, 2017.

[5] T. Chen, X. Xu, Y. Li, W. Wang, and L. Chen, "Speed-dependent coordinated control of differential and assisted steering for inwheel motor driven electric vehicles," Proceedings of the Institution of Mechanical Engineers, Part D: Journal of Automobile Engineering, 2017.

[6] R. Wang, C. Hu, Z. Wang, F. Yan, and N. Chen, "Integrated optimal dynamics control of 4WD4WS electric ground vehicle with tire-road frictional coefficient estimation," Mechanical Systems and Signal Processing, vol. 60, pp. 727-741, 2015.

[7] X. J. Jin, G. Yin, and N. Chen, "Gain-scheduled robust control for lateral stability of four-wheel-independent-drive electric vehicles via linear parameter-varying technique," Mechatronics, vol. 30, pp. 286-296, 2015.

[8] H. Qiu, Z. Dong, and Z. Lei, "Simulation and experiment of integration control of ARS and DYC for electrical vehicle with four-wheel-independent-drive," Journal of Jiangsu University (Natural Science Edition), vol. 37, no. 3, pp. 268-276, 2016.

[9] B. Li, H. Du, W. Li, and Y. Zhang, "Side-slip angle estimation based lateral dynamics control for omni-directional vehicles with optimal steering angle and traction/brake torque distribution," Mechatronics, vol. 30, pp. 348-362, 2015.

[10] R. Wang, H. Jing, C. Hu, F. Yan, and N. Chen, "Robust Ho Path Following Control for Autonomous Ground Vehicles With Delay and Data Dropout," IEEE Transactions on Intelligent Transportation Systems, vol. 17, no. 7, pp. 2042-2050, 2016.

[11] C. Hu, R. Wang, F. Yan, and N. Chen, "Output constraint control on path following of four-wheel independently actuated autonomous ground vehicles," IEEE Transactions on Vehicular Technology, vol. 65, no. 6, pp. 4033-4043, 2016.

[12] R. Wang, C. Hu, F. Yan, and M. Chadli, "Composite nonlinear feedback control for path following of four-wheel independently actuated autonomous ground vehicles," IEEE Transactions on Intelligent Transportation Systems, vol. 17, no. 7, pp. 2063-2074, 2016.

[13] B.-C. Chen and F.-C. Hsieh, "Sideslip angle estimation using extended Kalman filter," Vehicle System Dynamics, vol. 46, no. 1, pp. 353-364, 2008.

[14] L. Li, J. Song, H. Li, and X. Zhang, "A variable structure adaptive extended Kalman filter for vehicle slip angle estimation," International Journal of Vehicle Design, vol. 56, no. 1-4, pp. 161-185, 2011.

[15] B. L. Boada, M. J. L. Boada, and V. Diaz, "Vehicle sideslip angle measurement based on sensor data fusion using an integrated ANFIS and an Unscented Kalman Filter algorithm," Mechanical Systems and Signal Processing, vol. 72-73, pp. 832-845, 2016. 
[16] L. Li, G. Jia, X. Ran, J. Song, and K. Wu, "A variable structure extended Kalman filter for vehicle sideslip angle estimation on a low friction road," Vehicle System Dynamics, vol. 52, no. 2, pp. 280-308, 2014.

[17] Y.-H. Liu, T. Li, Y.-Y. Yang, X.-W. Ji, and J. Wu, "Estimation of tire-road friction coefficient based on combined APF-IEKF and iteration algorithm," Mechanical Systems and Signal Processing, vol. 88, pp. 25-35, 2017.

[18] K. Tin Leung, J. F. Whidborne, D. Purdy, and A. Dunoyer, "A review of ground vehicle dynamic state estimations utilising GPS/INS," Vehicle System Dynamics, vol. 49, no. 1-2, pp. 29-58, 2011.

[19] G. Baffet, A. Charara, and D. Lechner, "Estimation of vehicle sideslip, tire force and wheel cornering stiffness," Control Engineering Practice, vol. 17, no. 11, pp. 1255-1264, 2009.

[20] J. J. Rath, K. C. Veluvolu, M. Defoort, and Y. C. Soh, "Higherorder sliding mode observer for estimation of tyre friction in ground vehicles," IET Control Theory \& Applications, vol. 8, no. 6, pp. 399-408, 2014.

[21] F. Cheli, F. Braghin, M. Brusarosco, F. Mancosu, and E. Sabbioni, "Design and testing of an innovative measurement device for tyreroad contact forces," Mechanical Systems and Signal Processing, vol. 25, no. 6, pp. 1956-1972, 2011.

[22] H. Zhu, L. Li, M. Jin, H. Li, and J. Song, "Real-time yaw rate prediction based on a non-linear model and feedback compensation for vehicle dynamics control," Proceedings of the Institution of Mechanical Engineers, Part D: Journal of Automobile Engineering, vol. 227, no. 10, pp. 1431-1445, 2013.

[23] K. T. Leung, J. F. Whidborne, D. Purdy, and P. Barber, "Road vehicle state estimation using low-cost GPS/INS," Mechanical Systems and Signal Processing, vol. 25, no. 6, pp. 1988-2004, 2011.

[24] D. M. Bevly, J. Ryu, and J. C. Gerdes, "Integrating INS sensors with GPS measurements for continuous estimation of vehicle sideslip, roll, and tire cornering stiffness," IEEE Transactions on Intelligent Transportation Systems, vol. 7, no. 4, pp. 483-493, 2006.

[25] D. Piyabongkarn, R. Rajamani, J. A. Grogg, and J. Y. Lew, "Development and experimental evaluation of a slip angle estimator for vehicle stability control," IEEE Transactions on Control Systems Technology, vol. 17, no. 1, pp. 78-88, 2009.

[26] A. J. Tuononen, "Vehicle lateral state estimation based on measured tyre forces," Sensors, vol. 9, no. 11, pp. 8761-8775, 2009.

[27] A. K. Madhusudhanan, M. Corno, and E. Holweg, "Vehicle sideslip estimator using load sensing bearings," Control Engineering Practice, vol. 54, pp. 46-57, 2016.

[28] J.-H. Yoon and H. Peng, "A cost-effective sideslip estimation method using velocity measurements from two GPS receivers," IEEE Transactions on Vehicular Technology, vol. 63, no. 6, pp. 2589-2599, 2014.

[29] R. Wang and J. Wang, "Tire-road friction coefficient and tire cornering stiffness estimation based on longitudinal tire force difference generation," Control Engineering Practice, vol. 21, no. 1, pp. 65-75, 2013.

[30] L. Chen, M. Bian, Y. Luo, and K. Li, "Real-time identification of the tyre-road friction coefficient using an unscented Kalman filter and mean-square-error-weighted fusion," Proceedings of the Institution of Mechanical Engineers, Part D: Journal of Automobile Engineering, vol. 230, no. 6, pp. 788-802, 2015.

[31] X. Li, X. Song, and C. Chan, "Reliable vehicle sideslip angle fusion estimation using low-cost sensors," Measurement, vol. 51, no. 1, pp. 241-258, 2014.
[32] H. Zhang, X. Huang, J. Wang, and H. R. Karimi, "Robust energy-to-peak sideslip angle estimation with applications to ground vehicles," Mechatronics, vol. 2015, no. 30, pp. 338-347, 2014.

[33] J.-H. Yoon and H. Peng, "Robust vehicle sideslip angle estimation through a disturbance rejection filter that integrates a magnetometer with GPS," IEEE Transactions on Intelligent Transportation Systems, vol. 15, no. 1, pp. 191-204, 2014.

[34] J.-H. Yoon, S. Eben Li, and C. Ahn, "Estimation of vehicle sideslip angle and tire-road friction coefficient based on magnetometer with GPS," International Journal of Automotive Technology, vol. 17, no. 3, pp. 427-435, 2016.

[35] K. Maeda, H. Fujimoto, and Y. Hori, "Four-wheel drivingforce distribution method based on driving stiffness and slip ratio estimation for electric vehicle with in-wheel motors," in Proceedings of the 2012 IEEE Vehicle Power and Propulsion Conference, VPPC 2012, pp. 1286-1291, Republic of Korea, October 2012.

[36] D. Soffker, T.-J. Yu, and P. C. Muller, "State estimation of dynamical systems with nonlinearities by using proportionalintegral observer," International Journal of Systems Science, vol. 26, no. 9, pp. 1571-1582, 1995. 


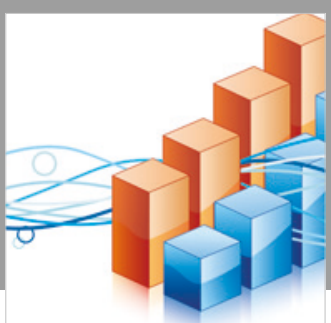

Advances in

Operations Research

\section{-n-m}
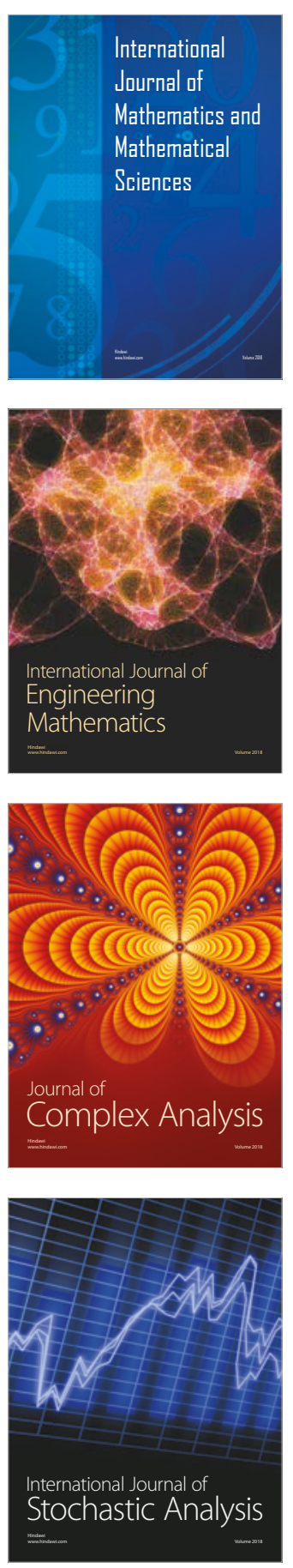
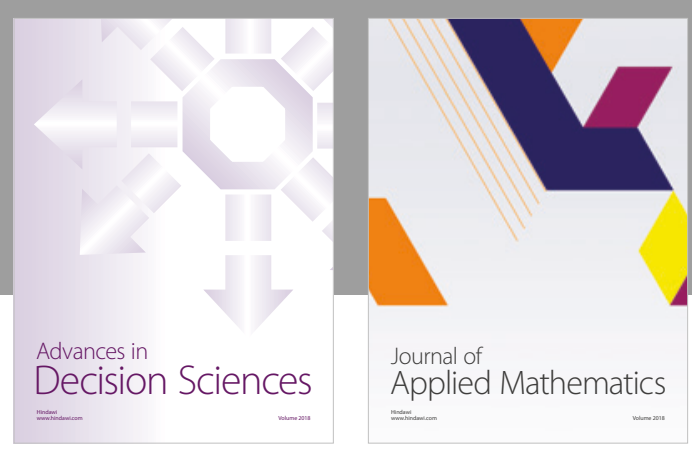

Journal of

Applied Mathematics
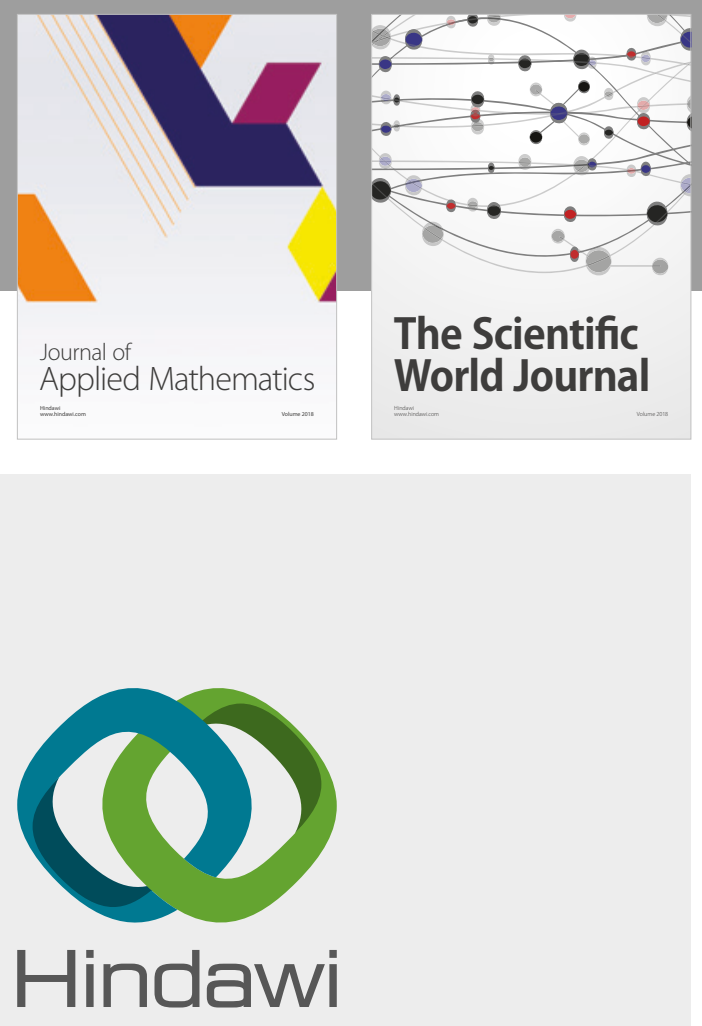

Submit your manuscripts at

www.hindawi.com

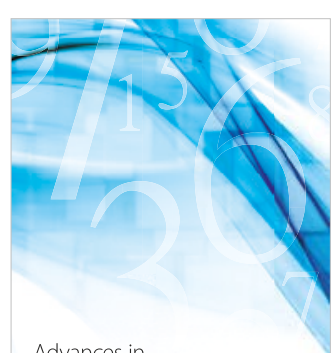

Advances in
Numerical Analysis
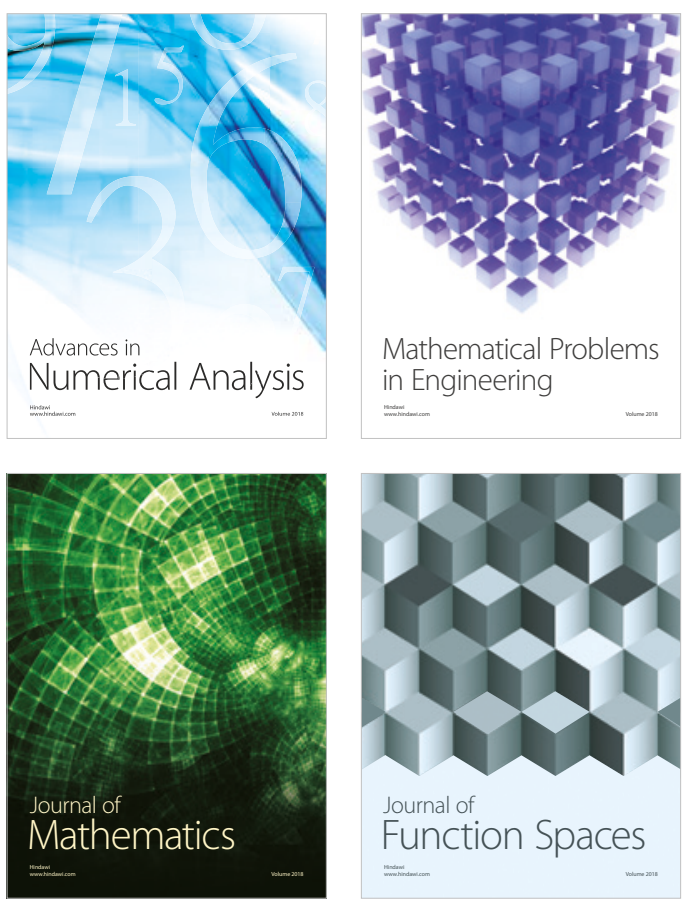

Mathematical Problems in Engineering

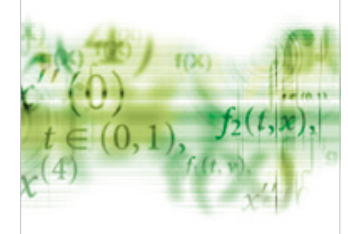

International Journal of

Differential Equations

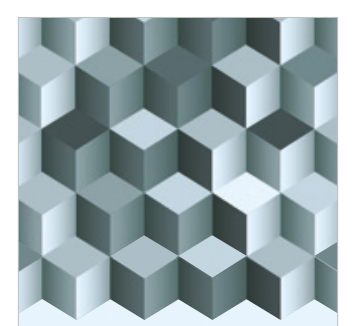

Journal of

Function Spaces

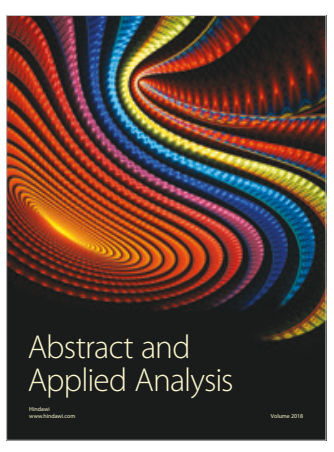

The Scientific

World Journal

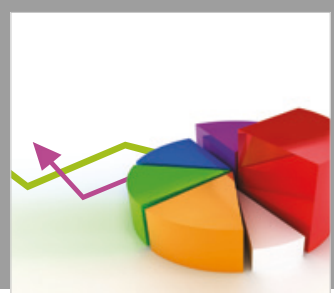

Journal of

Probability and Statistics
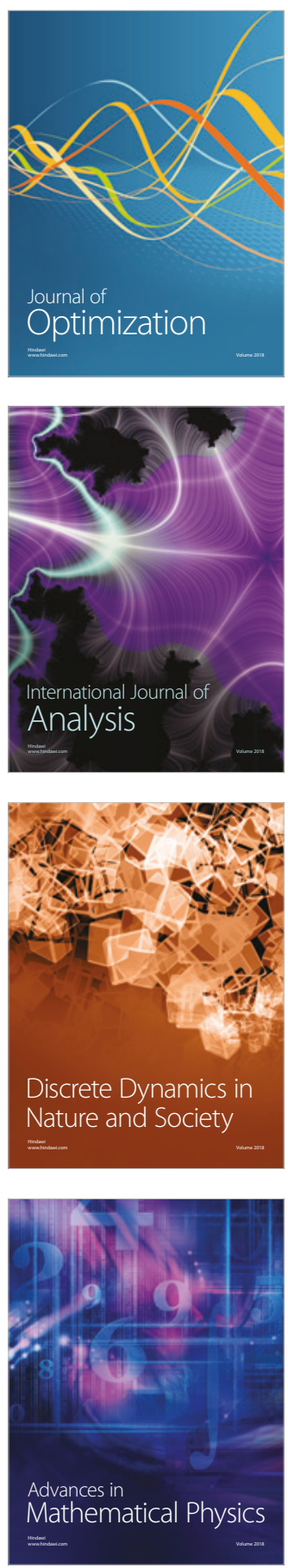